\title{
Combined Time of Flight and Photometric Stereo Imaging for Surface Reconstruction
}

\author{
Emma Le Francois ${ }^{1}$, Johannes Herrnsdorf ${ }^{1}$, Jonathan J. D. McKendry ${ }^{1}$, Laurence Broadbent ${ }^{2}$, Martin D. Dawson ${ }^{1}$ and Michael J. Strain $^{1}$ \\ ${ }^{1}$ Institute of Photonics, Department of Physics, SUPA, University of Strathclyde, Glasgow, UK \\ ${ }^{2}$ Aralia Systems, Bristol Robotics Laboratory, Bristol, UK \\ emma.le-francois@strath.ac.uk
}

\begin{abstract}
D reconstruction of objects can be achieved using both time of flight and photometric stereo imaging using four modulated white LEDs, a SPAD camera and a mobile phone. The standard deviation for the reconstruction is $4.1 \mathrm{~mm}$ at a distance of $70 \mathrm{~cm}$.
\end{abstract}

\section{Keywords - Time of Flight, Photometric Stereo, 3D surface reconstruction, LEDs, SPAD camera, Mobile device}

\section{INTRODUCTION}

Time-of-flight (ToF) and photometric stereo (PS) are three-dimensional (3D) imaging techniques with distinct, and complementary, areas of application. ToF imaging deals well with long range and discontinuous imaging but is limited by the resolution of current single photon camera systems or acquisition time of scanning systems. PS imaging uses conventional imagers and therefore has high spatial resolution but does not deal well with discontinuous surfaces. ToF is typically used for long-range light detection and ranging systems. A short-pulsed laser illuminates a scene in order to time correlate the reflected light intensity with the outgoing pulse to obtain a range map of the scene [1], and is commonly used in automotive and robotics applications. Alternatively, PS imaging is a passive method that relies on having one fixed camera perspective and different illumination directions to image an object in 3D [2]. This technique is more common in indoors scenarios for video surveillance, surface mapping and robot navigation [3]. Our previous work on "top-down" illumination PS imaging demonstrated 3D reconstruction with an error ranging from $3.5 \%$ to $10.4 \%$ for an object imaged at a distance of $42 \mathrm{~cm}$ [4]. However, this method used a black background to easily mask objects to reconstruct in order to speed up the computational reconstruction time. By employing a dual imaging system incorporating both ToF and PS the complementary properties of both systems can be used to image complex 3D fields with high resolution and complex discontinuities between objects.

Here we report the 3D reconstruction of an exemplar object (a sphere) using ToF - as a tool to mask the object, and PS imaging for a high-resolution surface reconstruction. A time-correlated single photon-counting (TCSPC) single photon avalanche diode (SPAD) camera is used with blue commercial light-emitting diodes (LEDs) to obtain a range map of the scene. For PS imaging, four white modulated LEDs illuminate the object while a mobile phone capture frames at 960 frames per second (fps). The LEDs are modulated at the camera frame rate with an orthogonal multiple access carrier schemes such that visible flicker is minimal, and no electronic synchronization is needed between the LEDs and the phone $[4,5]$. Our early results of the surface reconstruction show a root mean square error (RMSE) of $8.5 \%$.

\section{EXPERIMENT}

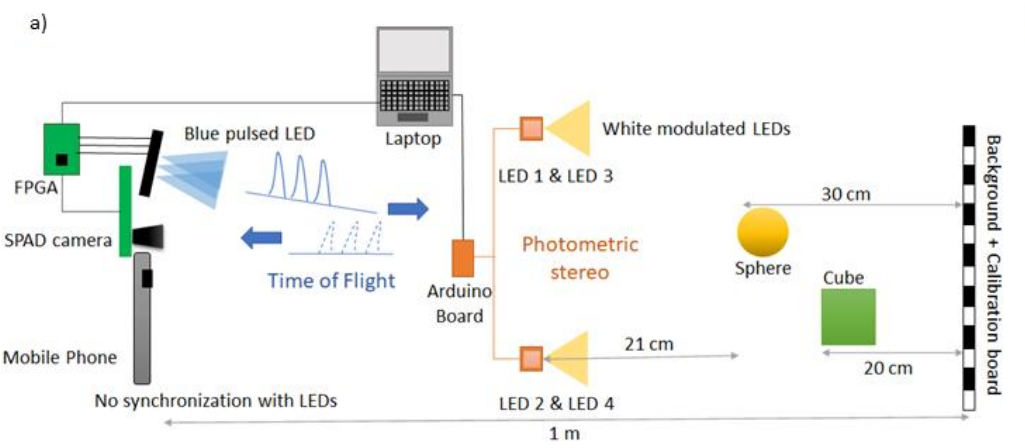

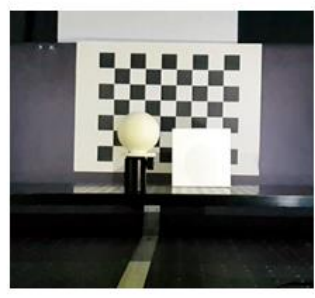

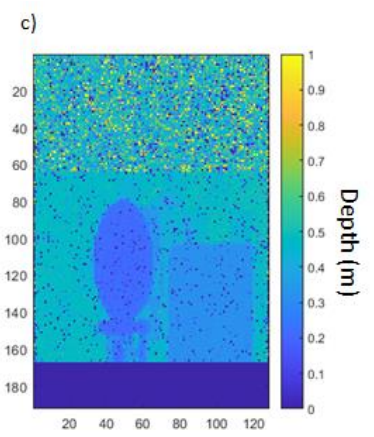

Fig. 1. a) Top-down schematic of the experimental setup, b) Cropped image taken with the mobile phone, c) Range Map of the scene using TCSPC mode with the SPAD camera

The experimental setup with both ToF and PS imaging is shown in Fig. 1.a). For ToF imaging, a SPAD image sensor is used and consists of a 192x128 SPAD pixels [5]. Each pixel is $18.4 \times 9.2 \mu \mathrm{m}^{2}$ in area and can be operated with TCSPC functionality, see [5,6] for more details. Both photon counting (PC) and TCSPC modes are needed for the calibration. PC mode is used to acquire the image 
intensity of a calibration board and the TCSPC mode for ToF, where time signal is provided by blue commercial LED array that is pulsed - $11.3 \mathrm{~ns}$ pulse wide at a repetition rate of $30 \mathrm{~ns}$ - with respect to a trigger signal from the SPAD camera. Both LED array and SPAD camera are controlled with field-programmable gate array (FPGA) modules. For PS imaging, four white commercial LEDs were placed $21 \mathrm{~cm}$ away from the object in a X shape. A mobile phone device (Samsung Galaxy 9) was mounted on a tripod as close as possible to the SPAD camera at a distance of $70 \mathrm{~cm}$ from the object with a field of view of 32 degrees. The phone captured frames with a resolution of $1280 \times 720$ at a rate of 960 frames per second (fps) for $0.2 \mathrm{~s} \mathrm{(see} \mathrm{picture} \mathrm{in} \mathrm{Fig.} \mathrm{1.b)).} \mathrm{For} \mathrm{the} \mathrm{illumination,} \mathrm{we}$ used an USB programmable controller board (Arduino Uno) to modulate the four LEDs at a frequency of $960 \mathrm{~Hz}$. Each LED was modulated with an individual multiple access carrier signal at a frequency of $960 \mathrm{~Hz}$, which is above visual flicker recognition and therefore suitable for digital lighting applications. The carriers were designed such that, analogous to orthogonal frequency division multiple access, no synchronization between the LEDs and the mobile phone was required $[4,5]$.

The SPAD intensity image was scaled and spatially registered to match the smartphone image dimensions using a checkerboard calibration object in the image. Then, a range map (Fig. 1.c)) obtained with the TCSPC mode is used to isolate the sphere by selecting its distance range. The subsequent PS imaging process carried out on the selected area follows the method from [4].

\section{RESULTS}
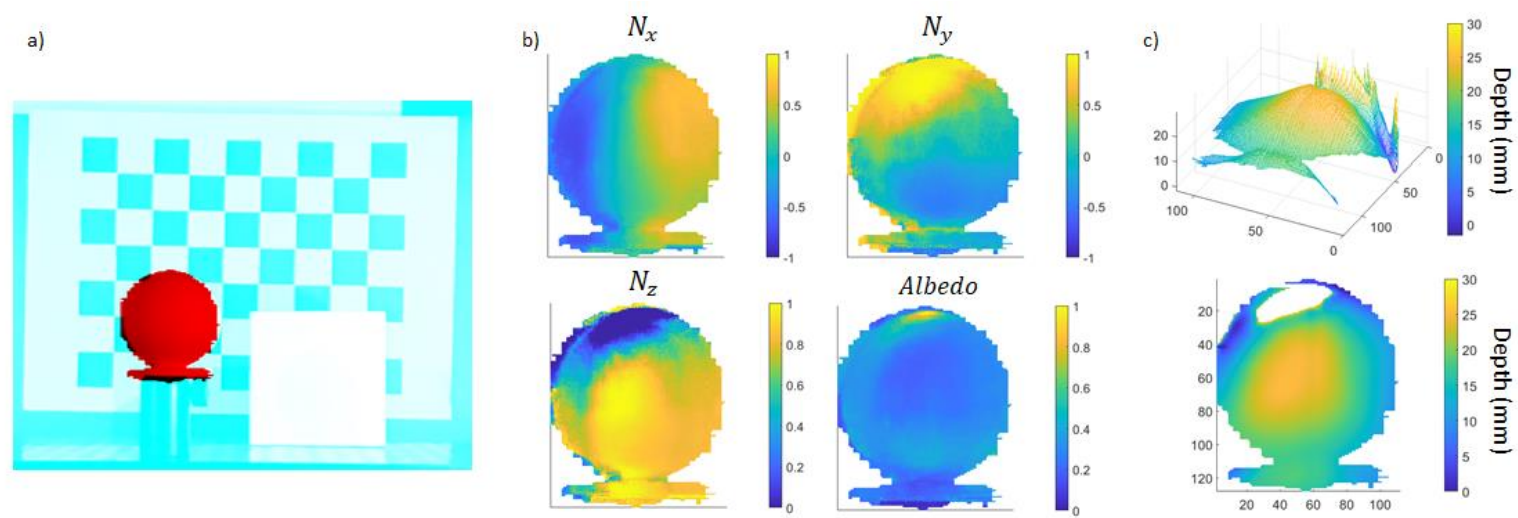

Fig. 2.a) ToF mask from SPAD camera superimpose on scene image, b) Surface normal components and Albedo of the sphere, c) 3D reconstruction of sphere.

Fig. 2.a) shows the ToF mask which is superimposed on the mobile phone image. We can see both cameras are correctly calibrated and aligned as the ToF mask coincides with the smartphone image of the sphere. Fig. 2.b) plots the surface normal components Nx, $\mathrm{Ny}$ and $\mathrm{Nz}$ of the sphere and its reflectivity (albedo). Nx and Ny correctly distinguishes left, right and up, down, respectively, facing surfaces of the object. As we cannot see the back of the sphere, $\mathrm{Nz}$ is always positive with some variations due to the depth of the object. The error apparent in Nz is likely due to the positionning of the four LEDs as the reflectivity is higher in this area, as shown in the albedo image. These surface normal components are used to calculate the surface topology with a reconstruction presented in Fig. 2.c). The error apparent in the Nz component clearly affects the top of the sphere reconstruction. Nonetheless, the global result is satisfactory and produces an RMSE of $4.1 \mathrm{~mm}$, which represents a normalized error of $8.5 \%$. This error is within our previous results [4] though the mobile phone is located at almost twice the distance in this setup.

\section{CONCLUSION}

Accurate calibration of a SPAD camera with a mobile phone has been demonstrated which gives the possibility to use ToF to improve our previous PS imaging technique. By using both ToF and PS imaging, we could simultaneously obtain depth information of a public area for example with a high-resolution 3D reconstruction of selected elements within the imaged scene.

\section{ACKNOWLEDGMENT}

We acknowledge support from the EPSRC (EP/M01326X/1, EP/T00097X/1). Emma Le Francois's studentship was part supported by Fraunhofer UK. We would also like to thank David Li for lending us the SPAD camera. DOI: https://doi.org/10.15129/0e4cc5fc-2f8e-4ff9-98bc-bead040ef5ac

\section{REFERENCES}

[1] M. Edgar, S. Johnson, D. Phillips, and M. Padgett, "Real-time computational photon-counting LiDAR,” Optical Engineering, vol. 57, no. 03, p. 1, 2017.

[2] R. J. Woodham, "Photometric Method For Determining Surface Orientation From Multiple Images," Optical Engineering, vol. 19, no. 1, pp. 139-144, 1980.

[3] A. Lipnickas and A. Kny, “A stereovision system for 3-d perception,” Elektronika ir Elektrotechnika, no. 3, pp. 99-102, 2009.

[4] E. L. Francois, J. Herrnsdorf, L. Broadbent, M. D. Dawson, and M. J. Strain, "Top-down illumination photometric stereo imaging using lightemitting diodes and a mobile device," in Frontiers in Optics + Laser Science APS/DLS, p. JTu3A.106, Optical Society of America, 2019.

[5] R. K. Henderson et al., "A $192 \times 128$ Time Correlated SPAD Image Sensor in 40-nm CMOS Technology,” IEEE J. Solid-State Circuits, vol. PP, pp. 1-10, 2019, doi: 10.1109/JSSC.2019.2905163.

[6] A. D. Griffiths et al., "Multispectral time-of-flight imaging using light-emitting diodes," vol. 27, no. 24, pp. 35485-35498, 2019. 